Oort: In spite of the many components one might expect the rotating matter to produce this absorption.

Kerr: This depends upon where the continuum source is in relation to the disk.

Oort: In connection with the tests for contraction or expansion of the Galaxy which Dr. Kerr mentioned, I should like to point to an investigation recently completed by Mr. Braes at Leiden. He used tracings made with the Dwingeloo telescope perpendicular to the galactic equator, in the region between $l^{I I}-5$ and $+5^{\circ}$. The outcome is that if there are general expansion or contraction effects they must be quite small, certainly less than $10 \mathrm{~km} / \mathrm{sec}$.

I would like to present some slides on the subject at a further session.

de Vaucouleurs: When we discuss expansion in the central regions we should keep in mind that the gas motion need not be isotropic in the plane.

Oort: What kind of gas? Spiral arms?

de Vaucouleurs : No, if there is any bar structure - even a weak one as in many galaxies one would rather expect any expansion motion to be mainly limited to gas streaming along the bar.

\title{
21. LARGE-SCALE STRUCTURE AND KINEMATICS OF THE GALACTIC SYSTEM
}

\section{B. LINDBLAD \\ Stockholms Observatorium}

It is, I believe, of fundamental importance to find out how far the structure and motions in the galaxies can be explained by gravitational theory. This does not deny that hydrodynamic and electromagnetic forces may play an important part. The special point which $I$ have in mind is the possibility, from the standpoint of gravitational theory, for the spiral structure to be a kind of quasi-steady state of comparatively long lifetime.

From the purely gravitational point of view we may expect that the spiral arms should be osculating to "dispersion orbits", that is orbits along which an association or a gaseous cloud has a natural tendency to disperse. An attempt to apply this criterion to the general spiral structure of the Galaxy has been made by B. Lindblad (1958). An application to the structure in the anticentre region has recently been made by Höglund (1963). Among more far-reaching attempts toward an interpretation of spiral structure may be mentioned the idea proposed by Aina Elvius (1958), later developed further by Aina Elvius and Herlofson (1960) and by Aina Elvius and P. O. Lindblad (1959), which considers the combined gravitational and electromagnetic force on a partially ionized gaseous ring and the effect of star formation releasing the electromagnetic force on the stellar matter. The gravitational theory of spiral structure has been developed extensively by P. O. Lindblad $(1960 a, b)$ who has shown by numerical computations that an ellipsoidal "dispersion ring" and bisymmetrical density waves in such a ring, which are proved to be in themselves steady phenomena, will produce perturbing effects by gravitational resonance leading to a development of spiral structure. This structure will in the first stages show "leading" arms with respect to the direction of rotation. At a later stage, however, these are transformed into arms of the "trailing" type. By the effects of differential rotation the arms will become successively more tightly wound, whereby the change 
in radius vector with the position angle about the centre will decrease. It does not seem, however, that this goes on indefinitely. Prolonged computations show that at a certain stage the spiral structure may be broken up across the arms in such a way that, passing certain stages of intermediate structures, the system will again develop a typical "trailing" arm structure with rather massive arms. In this way the spiral structure may be a quasi-periodic phenomenon, and the effective lifetime of spiral structures in a system may be appreciably prolonged.

It appears, however, that a rotating "trailing" arm system has certain properties which may work continuously in the same direction. Instead of a quasi-periodic spiral structure we may then get a quasi-steady formation of the spiral type (B. Lindblad 1963). The fundamental fact is here that a "trailing" arm in which the particles describe nearly circular motions about the centre has a great capacity for attracting and accumulating matter from the surroundings. This applies also to the mutual attraction between adjacent parts of different spiral arms. The situation is especially interesting in the case of the outermost parts of an arm which will have a tendency to fall in towards, and to become assimilated by, the neighbouring parts of the other spiral arm. It is important that these parts of the "inner" arm must expand owing to the attraction of the incoming matter. A quasi-steady state involving a kind of circulation between the outer parts of the arms will in this way be possible. There are several indications in galaxies of various types that such a circulation exists. I may mention here especially the galaxies M74, M83, M101, NGC 1300, and NGC 1566. In the last-mentioned very regular nebula there appears what may be called a double circulation with two wide branches returning towards the inner bright part of the spiral structure. In the regions close to the centre, effects will appear which tend to cut short the innermost spiral convolutions in a special process of circulation. This effect gives certain possibilities of an improved interpretation of the barred spiral structure. A quantitative examination of the circulation process in the outer regions leads to an estimate of the total mass of the spiral structure to be of the order of about $15 \%$ of the total mass of the system. A schematic picture of an ordinary spiral galaxy with returning branches appearing as "twigs" of the main spiral arms is shown here in Figure 1.

In the application to the Galactic System we fix our attention to the anticentre region, where deviations from purely circular motion are most easily detected. That the motion in low latitudes in the anticentre region is in the mean in-going is shown by results for the $21-\mathrm{cm}$ line from observations with the Dwingeloo telescope.

A diagram based on the early Leiden observations showing the maximum optical depth for the $21-\mathrm{cm}$ line in the interval of galactic latitude $-10^{\circ} \leqslant b^{\mathrm{I}} \leqslant+10^{\circ}$ as a function of galactic longitude and radial velocity shows, in addition to the regular features of the Cygnus-Carina arm and the Perseus arm, several branches of relatively strong inwards motion in the anticentre region. As has been mentioned above, Höglund (1963) has made a study of the velocity distribution in this region based on observations at the observatory of the Chalmers Technical University near Gothenburg. In the longitude-velocity diagram he has traced branches of obvious deviations from circular motion. In order to find the run of these branches in space he has tried 
to match the observations to osculating dispersion orbits. It seems that the pieces of matter thus obtained can largely be interpreted as branches in the sense mentioned above, proceeding inwards towards the region of the Perseus arm.

An important matter connected with the problems just mentioned concerns the composition of the spiral arms. It is probable according to the present theory that gas.clouds attracted by an arm will associate and assimilate with the gas clouds already in the arm. The interstellar clouds and the young OB-associations will therefore always constitute what may be called the "skeleton" of the arms. It is

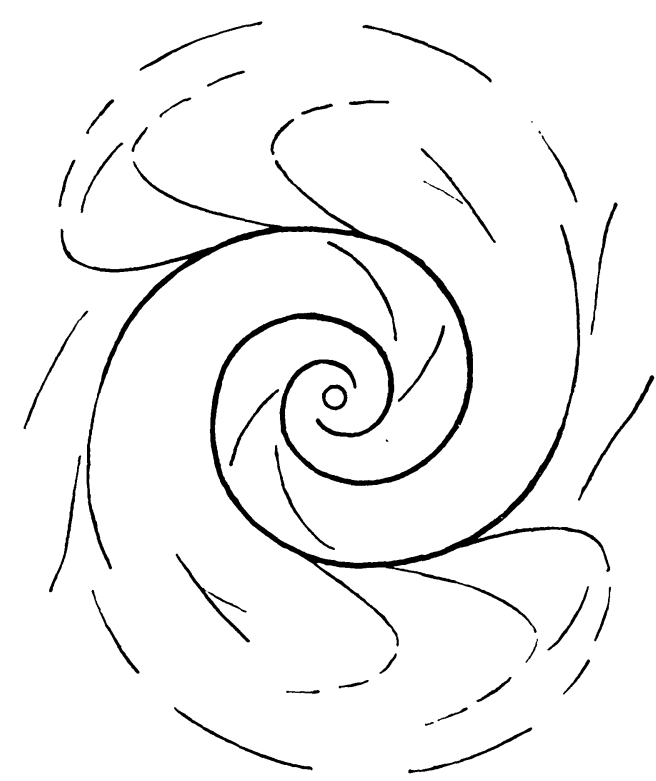

Fig. 1.-Schematic picture of an ordinary spiral galaxy with returning branches appearing as "twigs" of the main spiral arms.

probable, however, that the arms in a wider sense, corresponding to what we perceive as spiral arms on ordinary blue photographs of galaxies, are much more massive formations. In the photographs of spectra of galaxies taken by Mayall (1960) across the spiral structure the integrated spectra corresponding to the intersections with the arms appear to be of fairly late type with strong $H$ and $K$ lines and a strong G-band. As to our own Galaxy, Ramberg (1957) has investigated the composition of the spiral arm in the neighbourhood of the Sun in the Cepheus-Sagitta region. He finds for stars of type $A$ and for giants of types $G-K$ in this region a condensation in space which coincides nearly in distance with the arm defined by the Leiden observers by means of the $21 \mathrm{-cm}$ line of hydrogen. There is also a concentration of interstellar absorbing matter in the same region in space. A similar investigation has been made by Ramberg* in the luminous galactic cloud in Norma, with centre at galactic coordinates $l^{\mathrm{II}}=333^{\circ}, b^{\mathrm{II}}=-2^{\circ}$. He finds that in this direction space is

* Result kindly communicated by Professor Ramberg in advance of publication. 
practically free from interstellar absorption up to a distance of about $1050 \mathrm{pc}$, after which rather suddenly a marked excess of colour appears with a simultaneous increase of the space density of the stars. The depth of the cloud appears to be about $800 \mathrm{pc}$.

\section{References}

Elvius, A. (1958).-Pop. Astron. Tidskr. 39: 151.

Elvius, A., and Herlofson, N. (1960).-Ap.J. 131 : 304-9 (=Uppsala Obs. Medd. No. 128.).

Elvius, A., and Lindblad, P. O. (1959).-Arkiv Astron. 2: 393-406 (=Uppsala Obs. Medd.

No. 126.).

Höglund, B. (1963).-Arkiv Astron. 3: 215-72.

LindBLAD, B. (1958).-Stockholms Obs. Annaler 20 : No. 4.

LindBlad, B. (1963).-Stockholms Obs. Annaler 22: No. 5.

LindBlad, P. O. (1960a).-Stockholms Obs. Annaler 21 : No. 4.

Lindblad, P. O. (1960b).-Pop. Astron. Tidskr. 41 : 132-49.

Mayall, N. U. (1960).-Colloque Int. CNRS. [Paris 1959] Ann. d'Ast. 23: 344-59.

RAmberg, J. M. (1957).-Stockholms Obs. Annaler 20: No. 1.

\section{Discussion}

Bok: One should not overlook the fact that in many parts of the Southern Milky Way, the A stars are heavily concentrated in regions where there are not many OB stars or HII regions. The Carina region is a good example. One has a high density of A stars to a distance of 800 or $1000 \mathrm{pc}$ and no OB stars until $2000 \mathrm{pc}$ or a greater distance from the Sun.

Lindblad: Different constituents of the spiral arms will not always coincide. It is in fact not possible to argue a uniform composition of the arms. Great variation may occur and we should try to get information on the integrated composition. It seems that the dominating component as to spiral type can vary. In the actual cases the results cannot be doubted.

Buscombe: A good example is the prominent feature near Norma - the early $\mathrm{O}$ association for which Whiteoak from $U B V$ photometry finds $r=1400 \mathrm{pc}$, and Drs. Feast and Thackeray the mean radial velocity $-41 \mathrm{~km} / \mathrm{sec}$.

de Vaucouleurs: I should like to stress the importance of Dr. Lindblad's remarks on the inward-directed curl of the outer spiral arms in many galaxies. This is not a peculiarity but a normal feature in SB(s) systems, such as NGC 1300, where the arms seem to intersect without apparent disturbance. How is this possible if the arms contain gas? Or are they in different planes?

Lindblad: I am glad that you agree on the existence of the in-going branches. The condition of the outermost arms is complicated and the arms are rather broken up into pieces so that great variations may occur, probably also as to the plane. It seems that the importance of the returning matter for the dynamics of the system has not previously been fully realized.

\section{COMPARISON OF HI SPIRAL PATTERN WITH OPTICAL STRUCTURE OF OTHER GALAXIES}

\section{G. DE VAUCouleurs \\ University of Texas}

I. The maps of the spiral pattern of neutral hydrogen in the outer and intermediate regions of the Galaxy resulting from the $21 \mathrm{~cm}$ studies at Leiden and Sydney may be compared with the optical structure of other galaxies. If, as seems probable, most hydrogen arms coincide with or follow closely the optical arms marked by the blue supergiant stars, a comparison of the HI pattern with photographs in blue 